Tatout, C, Evans, D, Vanrobays, E, Probst, A and Graumann, K

The plant LINC complex at the nuclear envelope

Tatout, C, Evans, D, Vanrobays, E, Probst, A and Graumann, K (2014) The plant LINC complex at the nuclear envelope. Chromosome Research, 22 (2). pp. 241-252.

doi: 10.1007/s10577-014-9419-7

This version is available: https://radar.brookes.ac.uk/radar/items/37924ab7-429e-4163-8f9a-1416ef688d61/1/

Available on RADAR: April 2016

Copyright (C) and Moral Rights are retained by the author(s) and/ or other copyright owners. A copy can be downloaded for personal non-commercial research or study, without prior permission or charge. This item cannot be reproduced or quoted extensively from without first obtaining permission in writing from the copyright holder(s). The content must not be changed in any way or sold commercially in any format or medium without the formal permission of the copyright holders.

This document is the postprint version of the journal article. Some differences between the published version and this version may remain and you are advised to consult the published version if you wish to cite from it. 


\section{The plant LINC complex at the Nuclear Envelope}

2

3 Christophe Tatout ${ }^{1,3}$, David E. Evans ${ }^{2}$, Emmanuel Vanrobays ${ }^{1}$, Aline V. Probst ${ }^{1}$ and Katja Graumann ${ }^{2}$ 4

\section{6}

${ }^{1}$ Genetic reproduction and Development (GReD) - UMR CNRS 6293 - Clermont Université INSERM U 1103, 24 avenue des Landais, BP80026, 63171 Aubière Cedex, France, aline.probst@univ-bpclermont.fr, emmanuel.vanrobays@univ-bpclermont.fr, christophe.tatout@univ-
9

bpclermont.fr

${ }^{2}$ Department of Biological and Medical Sciences, Oxford Brookes University, Oxford OX3 0BP, United Kingdom, kgraumann@brookes.ac.uk, deevans@brookes.ac.uk ${ }^{3}$ corresponding author.

Running title: The plant LINC complex .

Keywords: Nuclear Envelope, LINC complex, SUN, KASH, nuclear shape

Summary: 187 words

Text without title page, abbreviations, summary and references: 5123 words

References: 63 
Abbreviations and definitions:

CDS: Cortical Division Site; The CDS is set up at the preprophase band and defines the attachment site of the cell plate during cytokinesis. The CDS determines the future division plane of the cell. CRWN: CRoWded Nuclei, formerly called Little Nuclei

ER: Endoplasmic Reticulum $\gamma$-TURC: $\gamma$-Tubulin Ring Complex

GIP: GCP3-Interacting Protein

INM: Inner Nuclear Membrane

IPNC: International Plant Nucleus Consortium

KASH: Klarsicht /Anc1/Syne1 Homology

LAD: Lamina Associated Domain

LINC: LInker of Nucleoskeleton and Cytoskeleton; The LINC complex is bridging the nuclear envelope and is made of KASH and SUN proteins.

MTOC: MicroTubule Organization Center; Plants lack centrosomes but instead develop nucleation centres of microtubules at the nuclear envelope during cell division.

NE: Nuclear Envelope; The NE is a double membrane surrounding chromatin.

NMCP: Nuclear Matrix Constituent Protein

MT: MicroTubule

NEBD: Nuclear Envelope Break Down

NES: Nuclear Export Signal

NLS: Nuclear Localization Signal

NPC: Nuclear Pore Complex; NPC are channels involved in trafficking between nucleus and cytosol and join INM and ONM.

ONM: Outer Nuclear Membrane.

PNS: Perinuclear Space at the Nuclear Envelope

PPB: Preprophase Band; The PPB is a dense ring of cortical microtubules that forms before the prophase of mitosis.

RanGAP: Ran GTPase-Activating Proteins

rER: rough Endoplasmic Reticulum

SUN: Sad1/Unc84

TM domain: trans-membrane domain

WIP: tryptophan $(\mathrm{W})$ - proline $(\mathrm{P})$ - proline $(\mathrm{P})$-Interacting Protein

WIT: WPP domain-Interacting Tail-anchored 
60 Significant advances in understanding the plant nuclear envelope have been made over the past few 61 years; indeed knowledge of the protein network at the nuclear envelope is rapidly growing. One such network, the Linker of Nucleoskeleton and Cytoskeleton (LINC) complex, is known in animals to connect chromatin to the cytoskeleton through the nuclear envelope. The LINC complex is made of Sad1/Unc84 (SUN) and Klarsicht/Anc1/Syne1 Homology (KASH) proteins which have been recently characterized in plants. SUN proteins are located within the inner nuclear membrane, while the KASH proteins are included into the outer nuclear membrane. SUN and KASH domains interact and bridge the two nuclear membranes. In Arabidopsis, KASH proteins also interact with the WPP domaininteracting tail-anchored protein 1 (WIT1), associated with the Nuclear Pore Complex and with myosin XI-I which directly interacts with the actin cytoskeleton. Although evidence for a plant LINC complex connecting the nucleus to the cytoskeleton is growing, its interaction with chromatin is still unknown but knowledge gained from animal models strongly suggests its existence in plants. Possible functions of the plant LINC complex in cell division, nuclear shape and chromatin organization are discussed. 


\section{An Overview of the Nuclear Envelope}

From the early beginning of research in genetics and cell biology, the nucleus has always been a center of great interest because it includes most of the genetic material of the eukaryotic cell. While its exact evolutionary origin remains uncertain, studies in Archaea, in which replication origins are attached to the cell periphery, suggest that the genetic material, as in prokaryotes, was originally attached to a membrane (Gristwood et al. 2012). To date, the origin of the nucleus cannot be resolved as a specific event in the course of evolution. Instead it is best explained by the coevolution of up to 27 cell components, including pre-existing endomembranes, which have surrounded the chromatin and created a specialized double membrane known as the Nuclear Envelope (NE) (Cavalier-Smith 2010).

At the structural level, the NE is made of a specialized Inner Nuclear Membrane (INM) and Outer Nuclear Membrane (ONM) separated by a Perinuclear Space (PNS) of about 25-30nm. The NE is in continuity with the Endoplasmic Reticulum (ER) and is interrupted by the nuclear pores (Figure 1A). Although the INM and ONM are continuous with each other and are fused together via the pore membrane, they contain varying sets of proteins to fulfill different functions. The ONM is studded with ribosomes similar to the rough ER (rER) and is involved in protein synthesis (Park and Blackstone 2010). The ONM binds microtubules (MTs) and can act as a nucleation centre of microtubules, which organize in Microtubule Organizing Centre (MTOC) at the basis of the mitotic spindle during cell division (Zhang and Dawe 2011; Masoud et al. 2013). In animals, the INM contains a set of proteins that interact with nuclear components including chromatin and the nucleoskeleton to keep a close association between NE and nuclear content (Starr 2009; Bickmore 2013). The part of the nucleoskeleton associated with the INM is also referred to as lamina since in animals it consists of lamin proteins. In plants, which lack lamin homologs, filamentous proteins are thought to form this scaffold, which is assembled into the so-called plamina (Fiserova et al. 2009; Fiserova and Goldberg 2010). Furthermore Dittmer et al. 2007 suggested that Arabidopsis CRoWded Nuclei (CRWN, formerly LIttle NuClei) and their plant homologs Nuclear Matrix Constituent Proteins (NMCP) may be part of the plamina based on their coiled-coil structures and their enriched localization at the nuclear periphery (Masuda et al. 1997; Dittmer et al. 2007; Ciska et al. 2013).

While the NE provides a physical separation of the genetic material into the nucleus to compartmentalize chromatin organization and function (Fransz and de Jong 2011), as well as protection against external damage (Gross and Bhattacharya 2011), it constrains communication between nucleus and the rest of the cell. This communication is integral to many vital processes. To overcome this problem, various structural features and properties of the nuclear periphery have evolved to allow exchange of molecules and signals as well as controlled access of cytoplasmic components to chromatin and therefore mediate communication and connectivity between the nucleus and the rest of the cell (Meier and Brkljacic 2009). Firstly, molecules such as proteins, RNA and charged ions can be exchanged between cytoplasm and nucleoplasm by specialized regions traversing 
the ONM and the INM formed by numerous Nuclear Pore Complexes (NPCs) (Tamura et al. 2010; Boruc et al. 2012). This highly regulated nucleo-cytoplasmic transport across NPC can either occur by passive diffusion (if the molecule is smaller than $40 \mathrm{KDa}$ ) or involve an active transport machinery (Keminer and Peters 1999). In the latter case, protein import from the cytosol into the nucleus then requires a Nuclear Localization Signal (NLS) within the protein sequence while export of proteins from the nucleus to the cytoplasm is mediated by nuclear export signal (NES). Additional nuclear transport factors such as RanGAP convey directionality to the protein exchange (Meier and Brkljacic 2009). Secondly, while import of proteins is functional in interphase, cell division also requires access of cytoplasmic components to chromatin. In open mitosis, this is accomplished by NE break down, where the NE membranes lose their associations with nuclear components and the chromatin is accessible to cytoplasmic molecules and structures such as the spindle microtubules (Evans et al. 2011).

A third form of communication between nucleus and cytoplasm takes place by signaling pathways. Ion channels and pumps are embedded in both INM and ONM and allow ions to be imported within the nucleoplasm. To date only a few of these channels and pumps have been studied, predominately those involved in $\mathrm{Ca}^{2+}$ signaling (Huda et al. 2013). Perinuclear calcium oscillations have been found to play important roles during symbiotic interactions and are thought to trigger a cascade of gene activation to help establish symbiotic interactions. While the Arabidopsis NE proteome still needs to be probed for ion channels and pumps, examples of these have been characterized in Lotus japonicus (Castor, Pollux), Medicago truncatula (Doesn't Make Infection 1, DMI1) and Solanum lycopersicum (Lycopersicon Ca(2+)-ATPase 1; LCA1) (Huda et al. 2013).

Finally, recent evidence suggests that physical protein networks bridge the membranes of the $\mathrm{NE}$, similar to non-plant systems, to link nuclear components and chromatin to cytoskeletal and cytoplasmic elements. Although these complexes are termed LInker of Nucleoskeleton and Cytoskeleton (LINC), they also connect chromatin and other non-skeletal proteins. In animals and fungi, however, their functions in nucleo-cytoskeletal linkage are better explored (Crisp et al. 2006). These NE bridges consist of INM and ONM intrinsic proteins that interact with each other in the periplasmic space. In plants, this area has received significant attention from research affording new insights into the composition and function of Arabidopsis LINC complexes. This review will aim to highlight the most recent advances with focus on LINC complex components and their roles in forming NE protein networks, nuclei shape, cell division and their potential implication in chromatin organization according to animal models.

\section{SUN and KASH domain proteins bridge the inner and the outer membrane of the NE}

LINC complexes involve specific protein components of the inner and outer nuclear membrane. At the INM, the proteins that take part in this linkage are termed Sad1/UNc84 (SUN) 
domain proteins, while the ONM proteins of the bridge are Klarsicht/Anc1/Syne1 Homology (KASH) domain proteins. To complete the linkage, SUN proteins recruit nuclear components to the bridges while KASH proteins interact with cytoplasmic components (Crisp et al. 2006; Starr 2011; Zhou and Meier 2013).

\section{SUN domain proteins}

The SUN domain proteins were defined according to their homology to Sad1 from Saccharomyces pombe and Unc-84 from Caenorhabditis elegans. SUN domain proteins are well conserved across all eukaryotes and share at least three distinct properties. They localize to the INM, contain one or more trans-membrane (TM) domains and a SUN domain (Starr 2009). The SUN domain proteins vary in size with the C. elegans UNC84 being one of the larger SUN proteins (1111aa), while yeast Sad1 and the AtSUN1/AtSUN2 are respectively 514, 471 and 455 aa in size (Haque et al., 2006; Graumann et al., 2010). The mechanisms needed to target the integration of SUN domain proteins within the INM are still largely unknown but according to data gained in yeast may rely on the TM domain, the NLS and specific factors targeting these membrane-anchored proteins (Schuldiner et al. 2008). SUN domain proteins can be divided into two sub-families according to the position of the SUN domain. Cter-SUNs contain a SUN domain at the C-terminal part of the protein while the mid-SUN proteins have a central (mid) SUN domain (figure 2A) (Graumann and Evans 2013a). In Arabidopsis, CterSUNs are the most studied although mid-SUNs were described in other organisms from yeast to human (Murphy et al. 2010; Sohaskey et al. 2010; Field et al. 2012; Friederichs et al. 2012). More recently, mid-SUN domain proteins AtSUN3, AtSUN4 and AtSUN5 were also identified in silico in Arabidopsis (Graumann and Evans 2013a) but their function still remains to be characterized.

Arabidopsis SUN1 and SUN2 are expressed in most tissues and organs (Graumann et al. 2010). The additional observation that double mutants severely effect reproduction (D.E and C.T unpublished data) suggests that the two are essential genes. From the $\mathrm{N}$ - to C-termini, these two proteins include at least four key features namely one NLS, a TM, a coiled-coil and a SUN domain (figure 2A). The TM domain allows Cter-SUN proteins to be anchored within the INM with the N-terminus located in the nucleoplasm, while the coiled-coil and SUN domain lie within the PNS (Graumann et al. 2010). Both Cter-SUNs localize at the NE (figure 2B) and mutations in Cter-SUNs were associated with nuclear shape modifications (figure 2C) (Zhou et al. 2012a). Their NE localization allows interactions with themselves through the coiled-coil domain and with KASH proteins through the SUN domain (Zhou et al. 2012a). Interaction between KASH and SUN domains implies that both domains are in close proximity to the periplasmic face of the ONM (Burke 2012). The mid-SUN domain proteins were first identified in maize as the plant-prevalent mid-SUN3 transmembrane (PM3) protein (Murphy et al 2010). In maize, ZmSUN3 and 4 mid-SUN proteins localise at the NE like Cter-SUN proteins, but distinct topology models have been proposed for Cter- and mid-SUN proteins (Murphy et al 2010). In 
contrast, in yeast, the Slp1 mid-SUN protein is located in the ER and was suggested to be involved in the NE localization of the Cter-SUN protein Mps3 (Friederichs et al. 2012). Whether the Arabidopsis mid-SUN proteins display similar ER localization to Slp1 or are true INM proteins as in maize remains to be investigated.

It is interesting to note that both sub-families of SUN domain proteins are well conserved across evolution (Murphy et al. 2010; Graumann et al. 2010; Field et al. 2012). Based on this high evolutionarily conservation, Cavalier Smith (2010) suggests their implication at the very beginning of NE formation by linking the NE with chromatin. It is also tempting to speculate that only Cter-SUNs may have the ability to interact with nucleoplasmic proteins through their N-terminal part while midSUNs display a TM domain too close to the ends of the protein leaving only a very short tail into the nucleoplasm (Figure 2A). As an example, the N-terminal domain of Sun-1 from Dictyostelium was shown by chromatin immunoprecipitation and electrophoretic mobility shift assay to bind chromatin and DNA, even though it does not contain any known DNA binding motif (Xiong et al. 2008). The modes of interaction between SUN domain proteins and chromatin still remain to be investigated in plants.

\section{KASH domain proteins}

The Klarsicht/Anc1/Syne1 Homology (KASH) domain proteins are C-tail anchored membrane proteins found at the ONM. The KASH domain is made of a TM domain followed by 6-30 amino acid residues and was first described in Caenorhabditis elegans (Starr and Han 2002). The KASH domain is positioned in the periplasm, which places the KASH domain close to the ONM. The amino acid sequence of the KASH domain is poorly conserved across kingdoms, apart from the penultimate amino acid being a proline, which is essential in mediating the SUN-KASH interactions (Sosa et al. 2012). In addition to the poorly conserved KASH domain, the proteins also vary tremendously in size - from the "small" Nesprin 4 of approximately $40 \mathrm{kDa}$ to the giant Nesprin 1 of $1000 \mathrm{kDa}$ (Razafsky and Hodzic, 2009). Starr (2009) proposed four criteria to define KASH domain proteins. First, KASH proteins are located at the ONM. Second, interaction between KASH and SUN requires the KASH domain. Third, the ONM localization is dependent upon interaction between the KASH domain and the SUN domain of SUN proteins (Crisp et al. 2006). The KASH domains are necessary and sufficient for localization of the KASH proteins at the ONM. Fourth, their N-terminal domain is not conserved and is located within the cytoplasm where many KASH proteins interact with components of the cytoskeleton such as actin and dynein.

Recently, KASH proteins have been identified in Arabidopsis. They are called WPP (tryptophan - proline - proline)-Interacting Protein (WIP) 1-3 (Zhou et al. 2012a) (figure 2A). These three proteins are plant-specific and meet the KASH domain characteristics mentioned - they are localized at the ONM (figure 2B), interact with the SUN domain and their ONM localization is 
dependent on the SUN-KASH interactions. The last four amino acids of their KASH domains are VVPT and thus include the cross-kingdom conserved penultimate proline. More importantly, this motif has homologs in various plant species indicating that this class of plant-specific VVPT-KASH proteins is conserved in plants (Zhou et al. 2012a; Zhou and Meier 2013). WIPs are able to interact with the plant-specific WPP domain of WPP containing proteins including RanGAP. Interestingly, deletion of Cter-SUN proteins causes loss of RanGAP association with the NE implying that in plants LINC complexes are involved in nucleo-cytoplasmic transport. Another family of proteins to associate with the WPP domain is the C-tail anchored WIT (WPP domain-Interacting Tail-anchored) protein family. WITs interact with WIPs (Zhao et al. 2008) and are thereby associated with plant LINC complexes. GFP-WIT1 localizes to the NE in a punctate (dotted) pattern indicative of an association with nuclear pores (Zhao et al. 2008). However interaction of WIT1 with NPC components has not been demonstrated so far. Interestingly, WIT1 also interacts with myosin XI-i connecting the SUNWIP-WIT complex with the actin cytoskeleton (Tamura et al. 2013). Finally, mutations in KASH domain proteins induce nuclear shape alterations very similar to those observed for SUN domain proteins (figure 2C) (Zhou et al. 2012a).

The function of WIP in RanGAP anchoring is specific to undifferentiated root tip cells (Xu et al., 2007) and it remains unclear which RanGAP anchoring mechanisms are in place in other tissues. However, tissue-specific expression of KASH proteins has also been observed in other higher eukaryotes like for the human KASH proteins called Nesprins (Sosa et al. 2013). This may suggest that other KASH proteins are likely to be discovered in plants in the future but this will remain challenging because of the poor conservation of the KASH domain.

\section{Toward the existence of a Plant LINC complex}

The core of a LINC complex is made by interaction between the Cter-SUN and the KASH domain proteins within the PNS. The structure has recently been resolved in animals using the human SUN2 (hSUN2) and the human KASH proteins Nesprin 1 and 2 (Zhou et al. 2012b; Sosa et al. 2012). hSUN2 organizes as trimers, a structure needed to allow hSUN2-KASH1/2 complexes to form. It is then expected that a trimeric SUN2 complex anchored at the INM binds three KASH proteins localized into the ONM. Homotrimers of hSUN2 form two distinct structures namely a stalk structure made by an $\alpha$-helix and a leaf-like structure formed by three $\beta$-sheet sandwiches (Zhou et al. 2012b; Sosa et al. 2012). The stalk structure of the hSUN2 SUN domain is indeed a short coiled-coil domain distinct from the classical larger coiled-coil domains found in most SUN domain proteins (see Sosa et al. 2012 for more details). It is linked to the leaf-like structure, which is critical for the SUN-KASH interactions (Zhou et al. 2012b; Sosa et al. 2012). In plants, both the SUN domain, which may also include the short coiled-coil region (Oda and Fukuda 2011), and the classical larger coiled-coil domain play a significant part in interactions among SUNs (Graumann et al. 2010). Furthermore, according to 
sequence alignments between SUN domains, key amino acid positions such as S641 within the SUN domain of hSUN2 are conserved in Arabidopsis SUN domain proteins and suggest LINC complexes in plants with similar organization.

So far, all studies on LINC complexes in animals have focused on Cter-SUN proteins as the INM components and only a yeast study has considered the putative role of the mid-SUN proteins. The interactions between Cter-SUNs and mid-SUNs would provide evidence that mid-SUNs are secondary components of the LINC complexes similar to WITs. With two Cter- and potentially three mid-SUN domain proteins (SUN1-5) located at the INM and at least three ONM components (WIP1-3), plant LINC complexes have the potential to be highly diverse in composition, available binding interactions and thus function. Some of these protein connections, as well as functions of plant LINC complexes, are presented below.

\section{Biological functions of plant LINC complexes}

The LINC complex has been proposed to play many different roles in animals such as connecting the nucleoskeleton and the cytoskeleton through the NE, anchoring telomeres at the INM to promote chromosome pairing, interacting with the centrosome during chromosome segregation or participating in nuclear migration during development or in response to stress (Starr 2009; Starr 2011). Many of these functions are still hypothetical in plants, but there is no doubt that the recent discovery of SUN and KASH proteins in plants will give the opportunity to study the role of the LINC complex in different aspects of nuclear function. Moreover, interactions between LINC components and putative nucleoskeletal as well as DNA-associated components are currently being studied.

\section{The plant LINC complex and cell division}

One striking outcome is the central properties of these NE components during mitosis in plants. Chromosome segregation during mitosis is driven by microtubules but unlike in animals, microtubules are not organized by centrosomes. Instead, numerous MT nucleation sites containing the conserved $\gamma$-Tubulin Ring complex ( $\gamma$-TURC) are found at the cell periphery, at MT branching points and the ONM (Masoud et al. 2013). The ONM is therefore instrumental in the formation of the mitotic spindle (Shaw et al. 2003). In addition to the MT nucleation sites, plants have developed other cell division - associated MT structures not found in animals and needed for cell division including the pre-prophase band (PPB) before mitosis and the phragmoplast after mitosis (Meier and Brkljacic 2009; Rasmussen et al. 2013) (figure 3A).

The formation of the pre-prophase band is one of the earlier events observed in the process of mitotic division. It marks the division plane and localizes where the new cell wall will be formed. As this MT-based structure and the NE break down, the spindle poles and spindle start to form (Meier and Brkljacic 2009; Rasmussen et al. 2013). Whether LINC complex components are involved in these 
processes is currently unknown. Localization of SUN1 and SUN2 in punctate structures at the beginning of mitosis might be an indicator of such involvement (figure 3). Also, it is known from animal and yeast systems that SUN and KASH proteins are involved in anchorage of centromeres. However, while in animal cells membranes are completely cleared from the mitotic spindle, in plants, membranes traverse the spindle (Irons et al. 2003; Graumann and Evans 2011). Interestingly Arabidopsis Cter-SUNs have been localized in these membranes (Graumann and Evans 2011) which also appear to stay in close proximity to the segregating chromosomes (figure 3 ). In animals, SUN proteins are one of the first INM proteins to re-associate with chromatin indicating they are important in NE reformation. Similarly in plants, Cter-SUNs and WIPs are observed to display a dynamic pattern during cell division. They first accumulate around chromatin next to the spindle pole, progress from the spindle pole to the cell plate to finally surround the chromatin forming the new NE (Graumann and Evans 2013b) (figure 3). A similar localization has been observed in dividing Apium graveolens (celery) and Allium cepa (onion) cells for the nucleoskeletal NMCP, which is a homolog to the Arabidopsis CRWN protein (Kimura et al. 2010; Ciska et al. 2013a). This kinetic of deposition may suggest that plant LINC complexes are involved in establishing local connections between chromatin and the reforming NE. However, NE-associated proteins that bind chromatin remain to be discovered. Finally, LINC complex components and other NE proteins have also been observed at the phragmoplast. This plant-specific structure consists of microtubules, microfilaments, and endoplasmic reticulum elements (figure 3 ). The microtubules and actin filaments within the phragmoplast serve to guide vesicles to build the new cell wall, which is seen at that stage between the two sister cells and known as cell plate. Whether the NE proteins are functional at the phragmoplast remains questionable. The non-functional NE marker LBR-GFP targets to the phragmoplast and the Arabidopsis Cter-SUN proteins are highly mobile in this structure while they are not in the NE indicating no specific interaction with other partners at the phragmoplast (Graumann et al. 2007; Graumann and Evans 2011).

\section{Plant LINC complexes and nuclear shape}

The nucleus has been considered by many researchers as a sphere sitting in the middle of the cell. However, it is now well documented that some tissues and organs display nuclei with various shapes, sizes and positions within the cell. Various plant model systems have been used to investigate these variations like root hairs, hypocotyls, pollen tubes and epidermis including trichomes (Traas et al. 1998; Chytilova et al. 2000). These variations in nuclear size and shape illustrate the dynamic or even elastic properties of the NE. Clearly modifications in the structure and protein interactions at the plant NE must occur to accommodate these changes but functions of NE components in these processes remains poorly understood. To date both SUN and KASH domain proteins are among the best-studied components of the plant NE. Currently, a new protein network is emerging at the NE. In 
addition to SUN1-5, KASH (WIP1-3), WIT1-2 and myosin XI-I, at least 30 nucleoporins assembled at the NPC have been identified (figure 4A).

To start addressing these questions, mutant analyses in Arabidopsis of some NE proteins including LINC complex proteins has highlighted the occurrence of similar phenotypes. sun1sun2 (Cte-SUN mutant), wiplwip2wip3 (KASH mutant) and kakul-1 (myosin XI-i mutant) alter nuclear shape in root hairs and trichomes in which elongated nuclei become more rounded (Zhou et al. 2012b; Tamura et al. 2013) (figure 4B). Conversely, over-expression of nup136, one of the plant nucleoporins, induces elongated nuclei in guard cells (Tamura and Hara-Nishimura 2011). A small nucleus phenotype was observed in crwnlcrwn2 (Dittmer et al. 2007). CRWN gene products are proposed to be associated with the plamina, the nucleoskeletal structure underlying the INM. These proteins are either core components of LINC complexes or associated with these, indicating that LINC complexes are essential in maintaining nuclear shape. In addition to shape, myosin XI-i is also responsible for mediating nuclear movement in root hairs as nuclei remain stationary in the mutant (Tamura et al. 2013). Alterations in nuclear shape very reminiscent to the "ghost-like" phenotype observed in animals for lamin mutants were also described for giplgip2 mutants (Janski et al. 2012). GIP1 and 2 (GCP3-Interacting Protein1 and 2) are involved in microtubule assembly at the NE by interacting with tubulin. This last observation may suggest that disruption of the microtubule cytoskeleton network leads to modifications in plant cell morphogenesis including nuclear shape as observed in the NE-associated mutants. The cytoskeleton is proposed to influence cell shape by guiding the deposition of new cell wall polymers. Correct positioning of the microtubules at the NE is also critical as in plants the NE is a microtubule organization center. Further investigations will be needed to demonstrate that microtubule disorganization is responsible for the rounded nuclei phenotype observed in NE-associated mutants.

\section{Future directions}

A new protein network including myosin XI-i, WIT, RanGAP, nucleoporins, KASH and SUN proteins is now emerging (figure 4A). As a common theme, nuclear shape alterations have been observed for most of the NE-associated mutants. Nuclear reshaping towards a more rounded shape may suggest the release of a structural constraint such as those exerted by the cytoskeleton and this in turn may alter gene expression by changing gene position within the nucleus (Versaevel et al. 2012). However, this has not been formally demonstrated except for interaction between the actin cytoskeleton and the myosin XI-i (Tamura et al. 2013). Other possible interactions with the cytoskeleton might involve microtubules. Indeed although the gip 1gip2 double mutant displays abnormal chromosome segregation with chromosome non-disjunction as expected for a component of the mitotic spindle, it also induces alteration in NE shape (Janski et al. 2012). Why do spindle associated proteins alter the nuclear shape? One attractive possibility would be that they are also part 
of the NE protein network. Other partners of the mitotic spindle such as MAD2, BUB1 and BUBR3-1 which have been recently reviewed (Masoud et al. 2013) could also be part of the NE protein network although to our knowledge no nuclear shape alterations have been recorded in mutant backgrounds for those candidates. Thus, to study protein interactions of GIP, MAD2, BUB3.1 and BUBR1 (Caillaud et al. 2009) with the NE-associated proteins described in this review will be of great interest. This may reveal ancestral relationship between nuclear and mitotic components as hypothesized by CavalierSmith 2010 and which may keep close together main components of the NE and chromatin during nuclear break down.

Still, connections between the nucleoskeleton and chromatin remain largely hypothetical in plants (figure 4A) while there is clear evidence in animals for interactions between the LINC complex and the chromatin components through the lamins (Haque et al. 2010; Yang et al. 2013). To date although there is no formal plant lamina, two lines of evidence argue in favor of its existence in plants (Ciska and Moreno Díaz de la Espina 2013). First, Fiserova et al. 2009 described filament structures connecting the NPC as well as $10 \mathrm{~nm}$ filaments within the nucleoplasm, two observations recalling the lamin structure in animals. Second, Arabidopsis and plant homologs NMCP/CRWN were suggested to be plant lamins based on their coiled-coil structures and their nuclear localization close to the NE (Masuda et al. 1997; Dittmer et al. 2007; Ciska et al. 2013). Demonstration of plant analogs of the lamins undoubtedly remains one of the key research issues on nuclear envelope during the next few years.

Lamins are associated with up to $40 \%$ of the total chromatin of the human genome defining the Lamina Associated Domain (LADs) (Bickmore 2013; Bickmore and van Steensel 2013). LADs have been also identified in Drosophila, where they localize close to the NE and are relatively genepoor. Genes at the lamina are transcriptionally silent and late replicating (Pickersgill et al 2006), characteristics common to heterochromatic domains. This suggests a more general role for the NE in maintenance of transcriptionally silent chromatin domains such as pericentric and centric heterochromatin (Vanrobays et al. 2013). These repetitive sequences can easily be visualized in interphase nuclei in Arabidopsis as they are grouped in compact domains of chromatin called chromocenters (Fransz et al. 2002). Thus it is expected that chromocenters will provide a valuable chromatin marker to investigate the possible interaction between the NE and heterochromatin by studying the possible effect of the NE-associated mutations described above on chromocenter features such as their position within the nucleus.

Today's research on the NE brings together research from cell biology and chromatin organization. Thanks to the International Plant Nucleus Consortium (IPNC) network formed in Salzburg in 2012 at the Society for Experimental Biology Annual Main Meeting, these two research communities are now actively collaborating to decipher the functions of the plant NE and its links to chromatin. 
We apologize for not having been able to quote all colleagues for their contribution in 402 deciphering the plant nuclear envelope network. K.G. is funded by an Early Career Fellowship from 403 the Leverhulme Trust. C.T, E.V. and A.V.P. are supported by the CNRS, INSERM, Blaise Pascal and 404 Auvergne Universities. A.V.P. is supported by ANR "Dynam'Het”ANR-11 JSV2 00901 and ANR 405 "SINODYN" ANR-12-ISV6-0001. C.T. and A.V.P are supported by the Region Auvergne through 406 "Life GRID" and a "Young Researcher Fellowship" respectively. 
Figure 1: The Plant Nuclear Envelope during interphase

$\mathrm{NE}$ is made of two distinct membranes (green) called the Outer Nuclear Membrane (ONM) and the Inner Nuclear Membrane (INM) forming the PeriNuclear Space (PNS). The ONM interacts with microtubules (MT) in random organization except during cell division where MTs grouped together as MicroTubule Organization Centers (MTOCs) to allow chromosome segregation (see also figure 3A). The NE is interrupted by numerous Nuclear Pore Complexes (NPCs; orange) and is connected with the rough Endoplasmic Reticulum (rER). The plamina (black) is the putative plant lamina. According to the animal models, NE, NPCs and plamina are expected to interact with chromatin (purple) within the nucleoplasm.

\section{Figure 2: SUN and KASH domain proteins}

421

A) Schematic representation of protein structures. Trans-membrane (TM), coiled-coil, SUN, WPP and KASH domains are shown. The KASH domain includes both the TM and the plant-specific VVPT motif (read arrow). B) Nuclear Envelope localization of GFP-AtSUN1 and CFP-AtWIP1 in leaves. Scale bar=10 $\mu \mathrm{m}$. C) Nuclear shape in SUN and KASH mutant backgrounds in leaf, trichome and vascular tissues. More rounded nuclei were observed in both mutant backgrounds and in all three tissues. Tissues were stained with ethidium bromide. Scale bar $=10 \mu \mathrm{m}$.

Figure 3: NE dynamics through the cell division

A) Keys features during plant cell division. Only chromatin (purple), NE and ER (green), microtubules (MT) (blue) and cell wall (brown) are indicated in this scheme. At the initiation of mitosis, chromatin compaction starts and MT are reorganized in an annular band called the PreProphase Band (PPB) just below the plasma membrane. The PPB disappears early during mitosis but leaves the Cortical Division Site (CDS) defining the future division plane of the cell. At the NE, MTs are also reorganized to the two opposite sides of the nucleus and formed MT Organization Centers (MTOCs). During the Nuclear Envelope Break Down (NEBD), spindles are connecting the chromosomes to ensure their segregation. During that stage, Cter-SUNs are observed in close proximity to the chromosome (green spheres). NE is reforming around chromatin first facing the spindle pole and finally proximal to the cell plate (green arrows). Finally, the cell plate (the newly formed cell wall) is formed along the phragmoplast (blue) before fusion between the new and parental cell wall. The cell plate is a disk-like structure bound at the CDS at cytokinesis and located at the center of the phragmoplast which is a cytoskeletal structure including microtubules and actin. 
442 B) SUN protein during cell division. BY2 cell expressing the SUN1-YFP (green) and the chromatin 443 marker histone H2B-CFP (magenta) through the cell cycle (redrawn from Graumann and Evans 444 2011). During the cell cycle SUN proteins remain always very close to chromatin. They accumulate in 445 the reforming NE at phragmoplast and cell plate. Scale bar $=10 \mu \mathrm{m}$.

446

$447 \quad$ Figure 4: Protein network at the Plant Nuclear Envelope

448 A) Protein interactions at the plant NE. Known interactions (green circles) involving SUN, KASH, 449 WIT, RanGAP, Myosin and Actin cytoskeleton as described in the text. Hypothesized interactions 450 (yellow circles) include WIT and other NPC components (nups), nups and SUN with the plamina as 451 well as nups, plamina and SUN with chromatin. B) Nuclear shape alterations in NE-associated 452 mutations. Pictures were reproduced with permission from Tamura et al. 2013 (kaku1-1), Zhou et al. 4532012 (wiplwip2wip3 and sun1sun2), Tamura and Hara-Nishimura 2011 (OEX-nup136) and Sakamoto 454 and Takagi 2013 (crwn1crwn2 also previously referred to as lincllinc2 in Dittmer et al 2007). 
457 Bickmore WA (2013) The Spatial Organization of the Human Genome. Annu Rev Genomics Hum 458 Genet 14:67-84.

459 Bickmore WA, van Steensel B (2013) Genome Architecture: Domain Organization of Interphase 460 Chromosomes. Cell 152:1270-1284.

461 Boruc J, Zhou X, Meier I (2012) Dynamics of the Plant Nuclear Envelope and Nuclear Pore. Plant 462 Physiol 158:78-86.

463 Burke B (2012) It Takes KASH to Hitch to the SUN. Cell 149:961-963.

464 Caillaud M-C, Paganelli L, Lecomte P, Deslandes L, Quentin M, Pecrix Y, Le Bris M, Marfaing N, 465 Abad P and Favery B (2009) Spindle Assembly Checkpoint Protein Dynamics Reveal Conserved and 466 Unsuspected Roles in Plant Cell Division. PLoS ONE 4:e6757.

467 Cavalier-Smith T (2010) Origin of the cell nucleus, mitosis and sex: roles of intracellular coevolution. 468 Biol Direct 5:7-7.

469 Chytilova E, Macas J, Sliwinska E, Rafelski SM, Lambert GM and Galbraith DW (2000) Nuclear 470 Dynamics in Arabidopsis thaliana. Mol Biol Cell 11:2733 -2741.

471 Ciska M, Masuda K and Moreno Díaz de la Espina S (2013) Lamin-like analogues in plants: the 472 characterization of NMCP1 in Allium cepa. J Exp Bot 64:1553-1564.

473 Ciska M and Moreno Díaz de la Espina S (2013) NMCP/LINC proteins: Putative lamin analogs in 474 plants? Plant Signal. Behav. 8:e26669.

475 Crisp M, Liu Q, Roux K, Rattner JB, Shanahan C, Burke B, Stahl PD and Hodzic D (2006) Coupling 476 of the nucleus and cytoplasm role of the LINC complex. J Cell Biol 172:41-53.

477 Dittmer TA, Stacey NJ, Sugimoto-Shirasu K, Richards EJ (2007) LITTLE NUCLEI genes affecting 478 nuclear morphology in Arabidopsis thaliana. Plant Cell 19:2793-2803.

479 Evans DE, Shvedunova M, Graumann K (2011) The nuclear envelope in the plant cell cycle: structure, 480 function and regulation. Ann Bot 107:1111-1118.

481 Field M, Horn D, Alsford S, Koreny L and Rout MP (2012) Telomeres, tethers and trypanosomes. 482 Nucleus 3:0-8.

483 Fiserova J, Kiseleva E, Goldberg MW (2009) Nuclear envelope and nuclear pore complex structure 484 and organization in tobacco BY-2 cells. Plant J 59:243-255.

485 Fiserova J, Goldberg MW (2010) Relationships at the nuclear envelope: lamins and nuclear pore 486 complexes in animals and plants. Biochem Soc Trans 38:829.

487 Fransz P, de Jong H (2011) From nucleosome to chromosome: a dynamic organization of genetic 488 information. Plant J 66:4-17. 
Fransz P, De Jong JH, Lysak M, Castiglione MR and Schubert I (2002) Interphase chromosomes in Arabidopsis are organized as well defined chromocenters from which euchromatin loops emanate. Proc Natl Acad Sci U S A 99:14584-14589.

Friederichs JM, Gardner JM, Smoyer CJ, Whetstine CR, Gogol M, Slaughter BD and Jaspersen SL (2012) Genetic Analysis of Mps3 SUN Domain Mutants in Saccharomyces cerevisiae Reveals an Interaction with the SUN-Like Protein Slp1. G3 Genes Genomes Genetics 2:1703-1718.

Graumann K, Irons SL, Runions J, Evans DE (2007) Retention and mobility of the mammalian lamin B receptor in the plant nuclear envelope. Biol Cell Auspices Eur Cell Biol Organ 99:553-562.

Graumann K, Runions J, Evans DE (2010) Characterization of SUN-domain proteins at the higher plant nuclear envelope. Plant J 61:134-144.

Graumann K, Evans DE (2011) Nuclear envelope dynamics during plant cell division suggest common mechanisms between kingdoms. Biochem J 435:661-667.

Graumann K, Evans DE (2013a) The nuclear envelope - structure and protein interactions. Plant Nucl. Struct. Genome Archit. Gene Regul. WILEY-BLACKWELL, pp 19-55

Graumann K, Evans DE (2013b) The nuclear envelope - Structure and protein interactions. Annu Plant Rev 46:19-56.

Gristwood T, Duggin IG, Wagner M, Albers SV and Bell SD (2012) The sub-cellular localization of Sulfolobus DNA replication. Nucleic Acids Res 40:5487-5496.

Gross J, Bhattacharya D (2011) Endosymbiont or host: who drove mitochondrial and plastid evolution? Biol Direct 6:12.

Haque F, Mazzeo D, Patel JT, Smallwood DT, Ellis JA, Shanahan CM and Shackleton S (2010) Mammalian SUN Protein Interaction Networks at the Inner Nuclear Membrane and Their Role in Laminopathy Disease Processes. J Biol Chem 285:3487 -3498.

Haque F, Lloyd DJ, Smallwood DT, Dent CL, Shanahan CM, Fry AM, Trembath RC and Shackleton S (2006) SUN1 Interacts with Nuclear Lamin A and Cytoplasmic Nesprins To Provide a Physical Connection between the Nuclear Lamina and the Cytoskeleton. Molecular and Cellular Biology 26: 3738-3751.

Huda KMK, Banu MSA, Tuteja R, Tuteja N (2013) Global calcium transducer P-type Ca2+-ATPases open new avenues for agriculture by regulating stress signalling. J Exp Bot 64:3099-3109.

Irons SL, Evans DE, and Brandizzi F (2003) The first 238 amino acids of the human lamin B receptor are targeted to the nuclear envelope in plants. J. Exp. Bot. 54: 943-950.

Janski N, Masoud K, Batzenschlager M, Herzog E, Evrard J-L, Houlné G, Bourge M, Chabouté M-E and Schmit A-C (2012) The GCP3-Interacting Proteins GIP1 and GIP2 Are Required for $\gamma$-Tubulin Complex Protein Localization, Spindle Integrity, and Chromosomal Stability. Plant Cell 24:11711187.

Keminer O, Peters R (1999) Permeability of Single Nuclear Pores. Biophys J 77:217-228. 
Kimura Y, Kuroda C, Masuda K (2010) Differential nuclear envelope assembly at the end of mitosis in suspension-cultured Apium graveolens cells. Chromosoma 119:195-204.

Masoud K, Herzog E, Chabouté M-E, Schmit A-C (2013) Microtubule nucleation and establishment of the mitotic spindle in vascular plant cells. Plant J 75:245-257.

Masuda K, Xu ZJ, Takahashi S, Ito A, Ono M, Nomura K and Inoue M (1997) Peripheral framework of carrot cell nucleus contains a novel protein predicted to exhibit a long alpha-helical domain. Exp Cell Res 232:173-181.

Meier I, Brkljacic J (2009) Adding pieces to the puzzling plant nuclear envelope. Curr Opin Plant Biol 12:752-759.

Murphy SP, Simmons CR, Bass HW (2010) Structure and expression of the maize (Zea mays L.) SUN-domain protein gene family: evidence for the existence of two divergent classes of SUN proteins in plants. BMC Plant Biol 10:269-269.

Oda Y, Fukuda H (2011) Dynamics of Arabidopsis SUN proteins during mitosis and their involvement in nuclear shaping. Plant J 66:629-641.

Park SH, Blackstone C (2010) Further assembly required: construction and dynamics of the endoplasmic reticulum network. EMBO Rep 11:515-521.

Pickersgill H, Kalverda B, de Wit E, Talhout W, Fornerod M, and van Steensel B (2006) Characterization of the Drosophila melanogaster genome at the nuclear lamina. Nat. Genet. 38: 10051014.

Rasmussen CG, Wright AJ, Müller S (2013) The role of the cytoskeleton and associated proteins in determination of the plant cell division plane. Plant J 75:258-269.

Razafsky D, and Hodzic D (2009) Bringing KASH under the SUN: the many faces of nucleocytoskeletal connections. J Cell Biol 186: 461-472.

Schuldiner M, Metz J, Schmid V, Denic V, Rakwalska M, Schmitt HD, Schwappach B and Weissman JS (2008) The GET Complex Mediates Insertion of Tail-Anchored Proteins into the ER Membrane. Cell 134:634-645.

Shaw SL, Kamyar R, Ehrhardt DW (2003) Sustained Microtubule Treadmilling in Arabidopsis Cortical Arrays. Science 300:1715-1718.

Sohaskey ML, Jiang Y, Zhao JJ, Mohr A, Roemer F and Harland RM (2010) Osteopotentia regulates osteoblast maturation, bone formation, and skeletal integrity in mice. J Cell Biol 189:511-525.

Sosa BA, Kutay U, Schwartz TU (2013) Structural insights into LINC complexes. Curr Opin Struct Biol 23:285-291.

Sosa BA, Rothballer A, Kutay U, Schwartz TU (2012) LINC Complexes Form by Binding of Three KASH Peptides to Domain Interfaces of Trimeric SUN Proteins. Cell 149:1035-1047.

Starr DA (2009) A nuclear-envelope bridge positions nuclei and moves chromosomes. J Cell Sci 122:577-586. 
Starr DA, Han M (2002) Role of ANC-1 in Tethering Nuclei to the Actin Cytoskeleton. Science 298:406-409.

Tamura K, Fukao Y, Iwamoto M, Haraguchi T and Hara-Nishimura I (2010) Identification and Characterization of Nuclear Pore Complex Components in Arabidopsis thaliana. Plant Cell Online 22:4084-4097.

Tamura K, Hara-Nishimura I (2011) Involvement of the nuclear pore complex in morphology of the plant nucleus. Nucleus 2:168-172.

Tamura K, Iwabuchi K, Fukao Y, Kondo M, Okamoto K, Ueda H, Nishimura M and Hara-Nishimura I (2013) Myosin XI-i Links the Nuclear Membrane to the Cytoskeleton to Control Nuclear Movement and Shape in Arabidopsis. Curr Biol 23: 1776-1781.

Traas J, Hülskamp M, Gendreau E, Höfte H (1998) Endoreduplication and development: rule without dividing? Curr Opin Plant Biol 1:498-503.

Vanrobays E, Thomas M, Tatout C (2013) Heterochromatin positioning and Nuclear Architecture. Plant Nucl. Struct. Genome Archit. Gene Regul. WILEY-BLACKWELL, pp 157-190

Versaevel M, Grevesse T, Gabriele S (2012) Spatial coordination between cell and nuclear shape within micropatterned endothelial cells. Nat Commun 3:671.

Xiong H, Rivero F, Euteneuer U, Mondal S, Mana-Capelli S, Larochelle D, Vogel A, Gassen B and Noegel AA (2008) Dictyostelium Sun-1 Connects the Centrosome to Chromatin and Ensures Genome Stability. Traffic 9:708-724.

Xu XM, Meulia T and Meier I (2007) Anchorage of Plant RanGAP to the Nuclear Envelope Involves Novel Nuclear-Pore-Associated Proteins. Current Biology 17: 1157-1163.

Yang L, Munck M, Swaminathan K, Kapinos LE, Noegel AA and Neumann S (2013) Mutations in LMNA Modulate the Lamin A - Nesprin-2 Interaction and Cause LINC Complex Alterations. PLoS ONE 8:e71850.

Zhang H, Dawe RK (2011) Mechanisms of plant spindle formation. Chromosome Res 19:335-344. Coiled-Coil Proteins Are Required for the Tissue-Specific Nuclear Envelope Targeting of Arabidopsis RanGAP. Plant Cell Online 20:1639-1651.

Zhou X, Graumann K, Evans DE, Meier I (2012a) Novel plant SUN-KASH bridges are involved in RanGAP anchoring and nuclear shape determination. J Cell Biol 196:203-211.

592 Zhou Z, Du X, Cai Z, Song X, Zhang H, Mizuno T, Suzuki E, Yee MR, Berezov A, Murali R, Wu SL, 593 Karger BL, Greene MI and Wang Q (2012b) Structure of Sad1-UNC84 Homology (SUN) Domain 594 Defines Features of Molecular Bridge in Nuclear Envelope. J Biol Chem 287:5317-5326. 
Figure 1

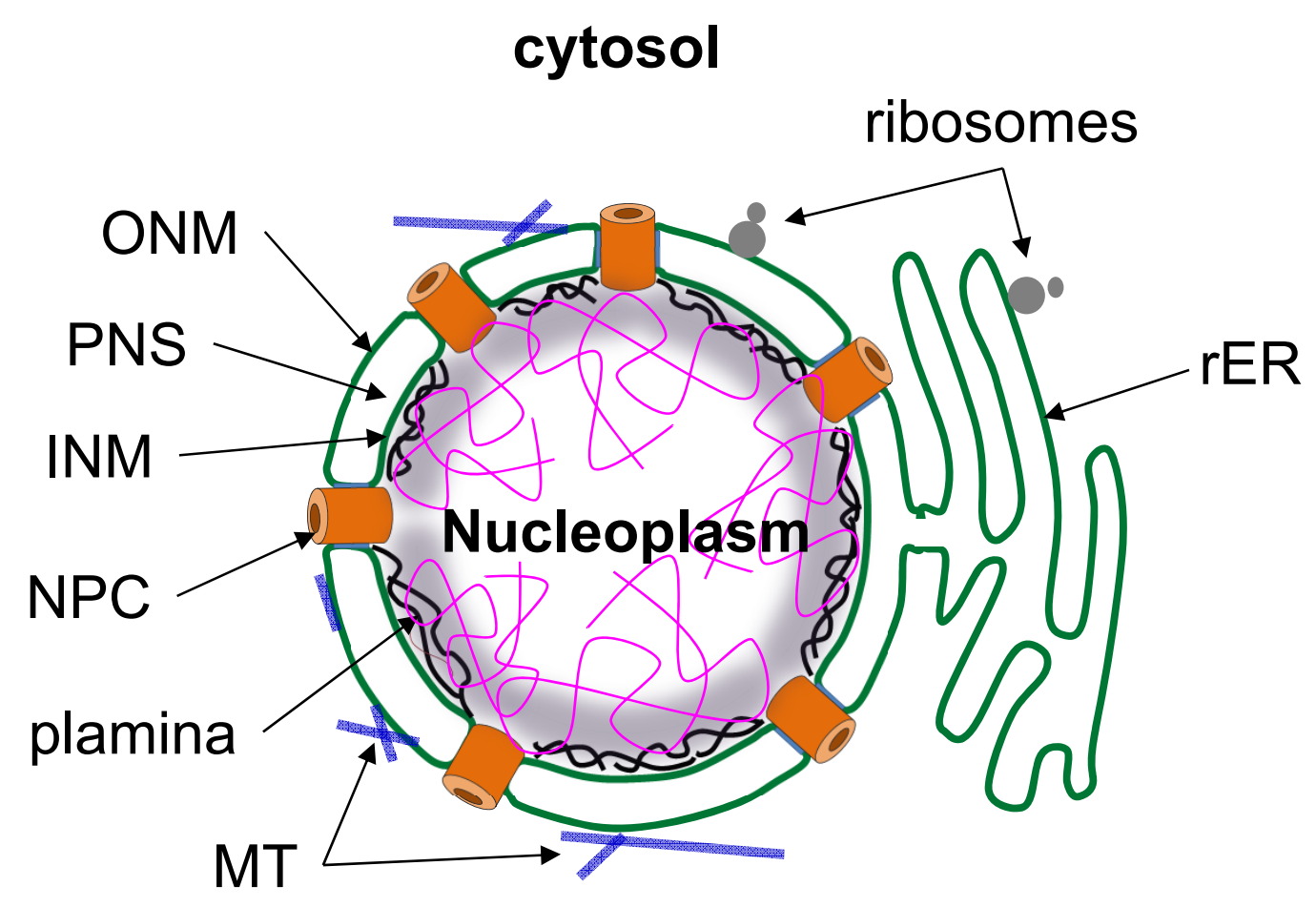


Figure 2

A
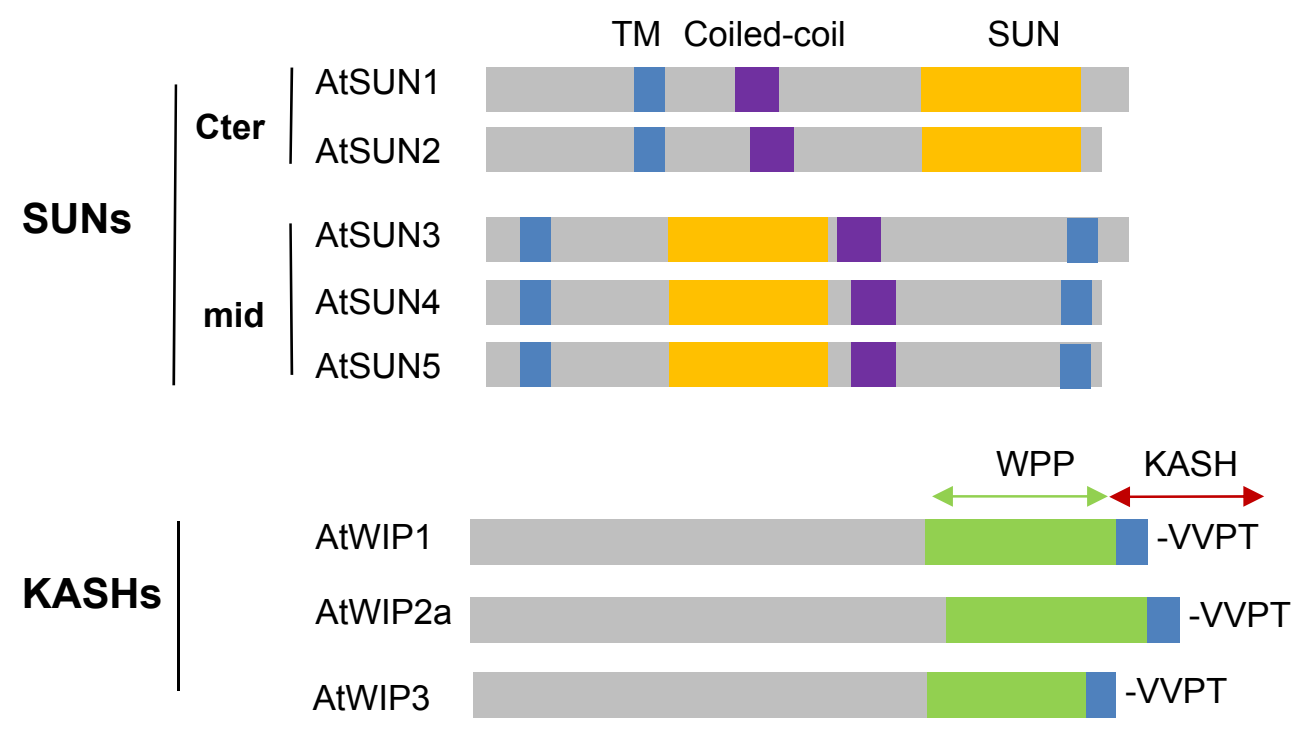

B

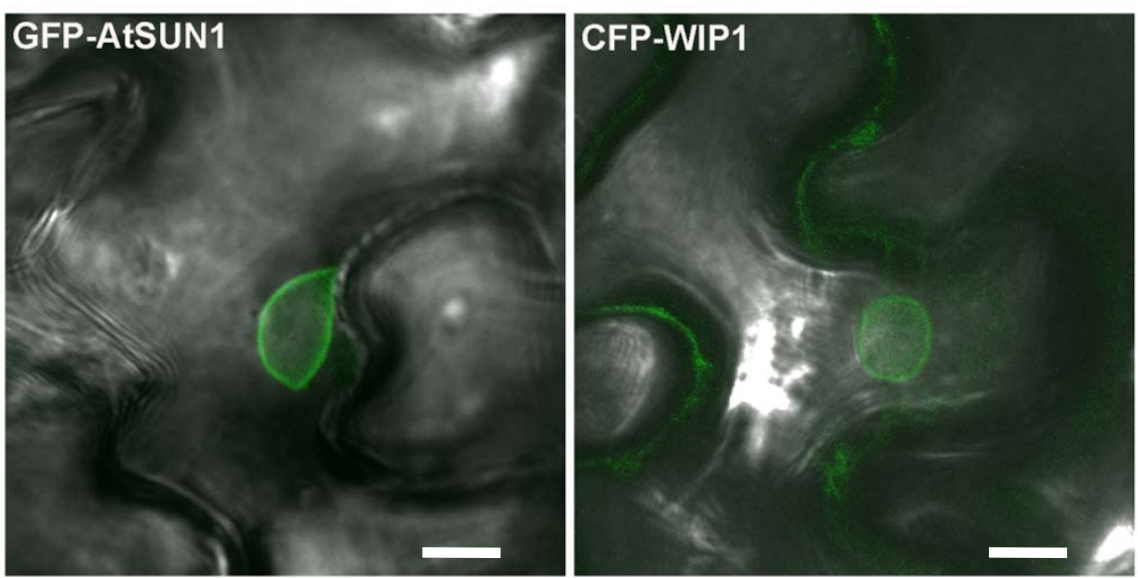

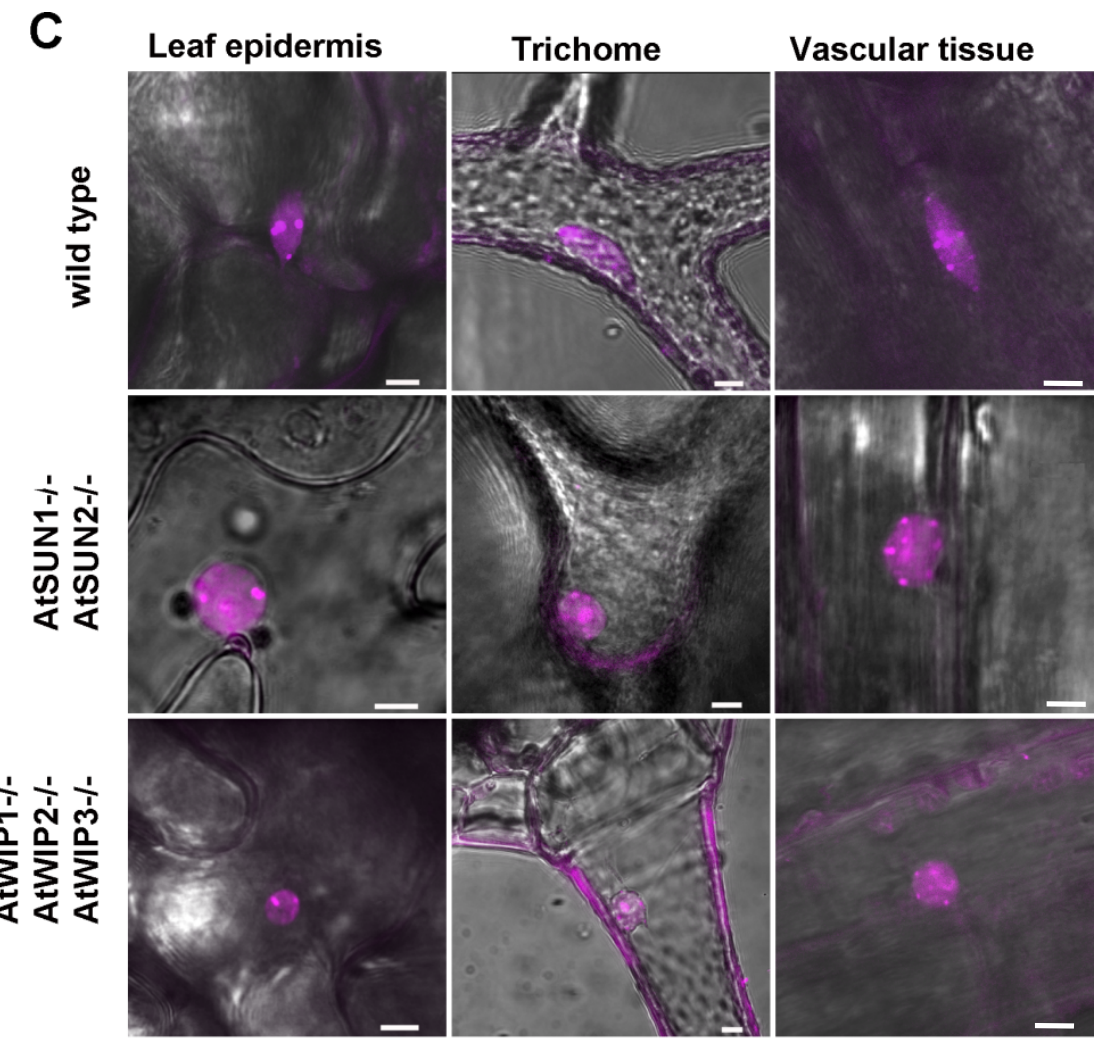


Figure 3

A
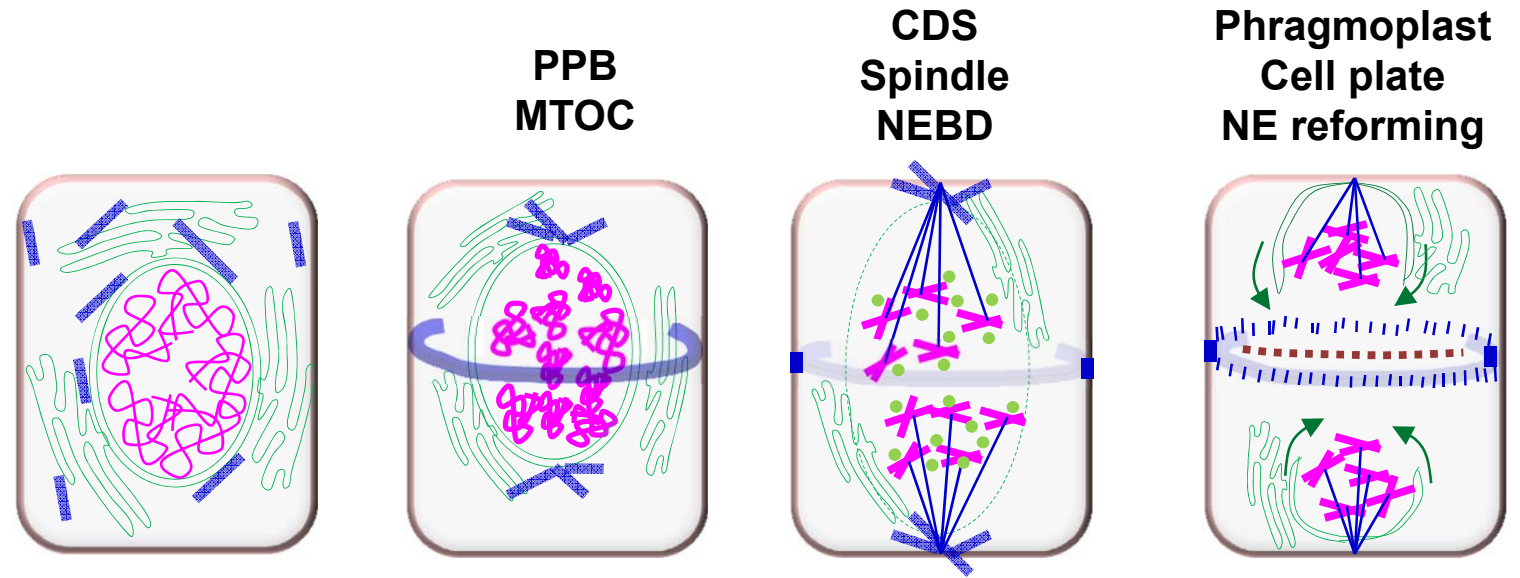

Cell wall fusion

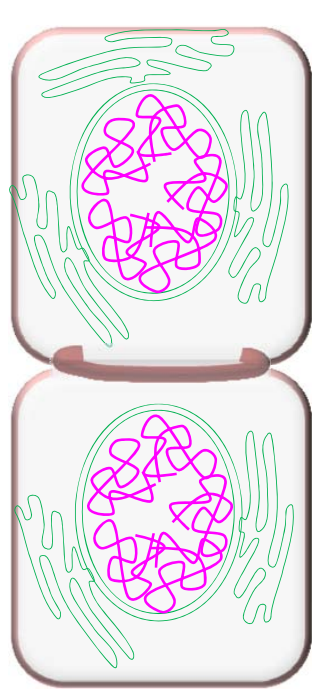

B Interphase

Prophase

Prometaphase

telophase

Cytokinesis
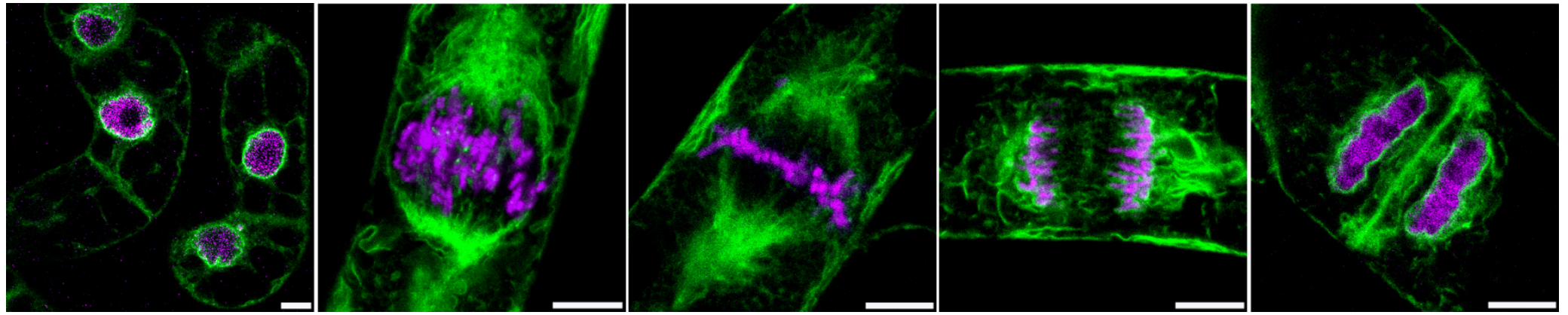
Figure 4

A

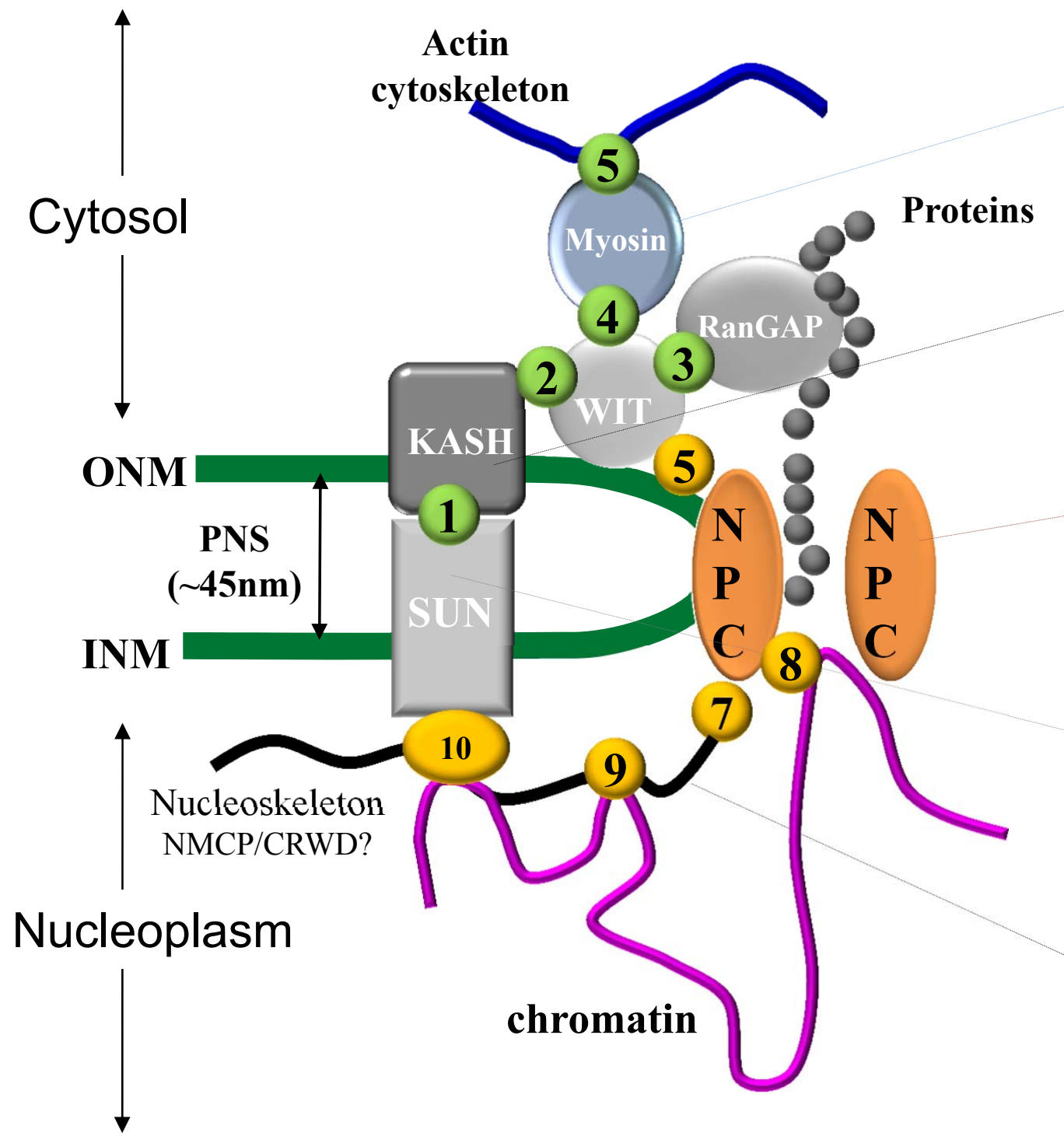

B

wt mutants

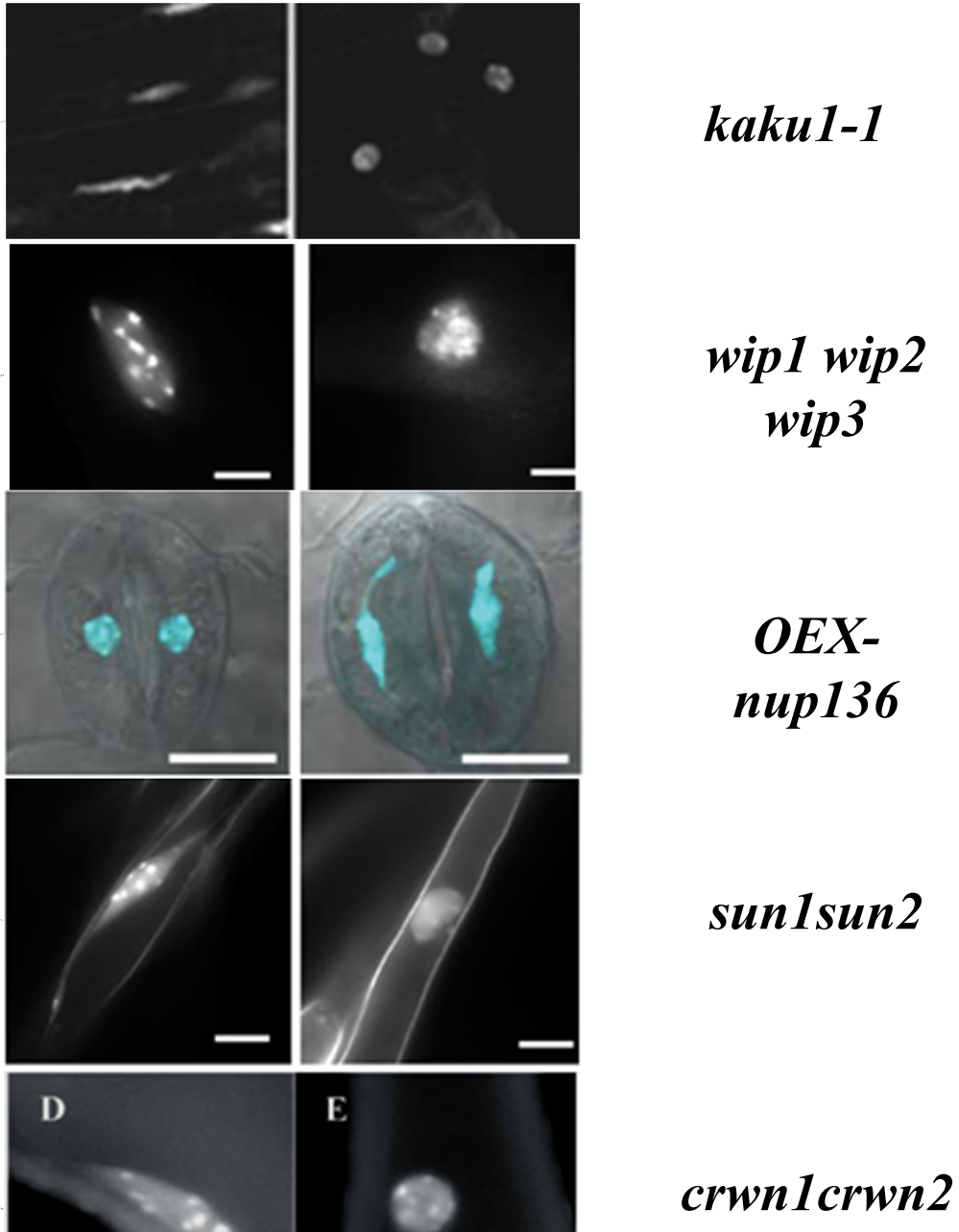

